

\section{A novel, multidisciplinary clinic for complex visual problems in older people}

${ }^{1}$ Newcastle Eye Centre, Royal Victoria Infirmary, Newcastle upon Tyne, UK

${ }^{2}$ Institute for Ageing and Health, Newcastle

University, Newcastle upon Tyne, UK

${ }^{3}$ Newcastle Older Person Services, Northumberland Tyne and Wear NHS

Foundation Trust, Newcastle upon Tyne, UK

${ }^{4}$ Department of Neurology, Sunderland Royal Hospital, Kayall Road, Sunderland, UK

${ }^{5}$ Institute of Neurosciences, Newcastle University, Newcastle upon Tyne, UK

Correspondence: JM Jefferis, Clinical Ageing Research Unit, Campus for ageing and vitality, Newcastle University, Newcastle Upon Tyne, NE4 5PL, UK

Tel: +44 (0)191 248 1297;

Fax: +44 (0)191248 1250

E-mail: Joanna.jefferis@

ncl.ac.uk

Received: 1 August 2012 Accepted in revised form: 26 August 2012

Published online:

12 October 2012

\begin{abstract}
Background Visual symptoms in older people can be complex and inadequately explained by eye pathology alone. Psychological and neurodegenerative processes may manifest as complex visual symptoms, and thus some patients may be poorly served by a purely ophthalmic approach. We have developed a novel multidisciplinary clinic with input from neurology, ophthalmology, and psychiatric specialists. Here, we describe the patient population, disease prevalence, and potential impact of this new clinic.

Methods A retrospective audit of paper and electronic records from June 2010 to February 2012 and selected case reports.

Results Between June 2010 and February 201248 patients attended the clinic. Notes were available for $47(98 \%)$. Mean age was 76.2 (range 48-92). The main presenting complaints were hallucinations, followed by nonspecific visual deficit, double vision, blurred vision, and visuospatial deficit. Cognitive impairment was noted in $68 \%$ $(32 / 47)$ of patients, of which $16 / 32(50 \%)$ were new diagnoses. We were able to give a diagnosis to $98 \%$ (46/47) of patients; of these, $74 \%(35 / 46)$ were new diagnoses. A total of $6 \%(3 / 47)$ were felt to have presentations attributable to eye pathology alone, whereas $89 \%(42 / 47)$ were felt to have a neuropsychiatric component. Management included referral to other clinics for continuing care in $43 \%(20 / 47)$ and initiation of therapy in $36 \%$ (17/47). The three case reports demonstrate cases, where our multidisciplinary approach aided diagnosis and management of patients with complex visual symptoms.

Conclusion A combined clinic with neurological, ophthalmic, and psychiatric
\end{abstract}

RC Han', JM Jefferis ${ }^{1,2}$, JP Taylor ${ }^{2,3}$, NK Archibald ${ }^{4}$ and MP Clarke ${ }^{1,5}$

input is an effective way to diagnose and manage complex visual problems in older people.

Eye (2012) 26, 1536-1541; doi:10.1038/eye.2012.205; published online 12 October 2012

Keywords: visual perception; dementia; neurodegenerative disease; multidisciplinary communication; aging

\section{Introduction}

Eye pathology is increasingly common with advancing age and frequently coexists with other morbidity in the older patient population. ${ }^{1,2}$ Complex visual symptoms in such patients may be inadequately explained by eye pathology alone. ${ }^{2-4}$ Neurodegenerative disease, psychotropic medications, and psychological factors may all manifest with or exacerbate symptoms of visual dysfunction. ${ }^{5-14}$ Such multifactorial visual complaints are difficult to diagnose, and may be best approached by a multidisciplinary team. ${ }^{3}$

The Visual Perception Clinic in the Newcastle Eye Centre provides such a multidisciplinary service. The clinic adheres to the following format: (1) Multidisciplinary discussion of each case from the referral letter by clinicians from ophthalmology, old-age psychiatry, and neurology followed by designation of a clinical lead. (2) History and examination by the clinical lead with additional clinical evaluation or investigations as indicated, which may include cognitive assessment, refraction, orthoptic assessment, and imaging. (3) Further multidisciplinary discussion and formulation of management plan, which is relayed back to the patient by the team. The clinic runs in the Eye Department with support from optometrists, orthoptists, and radiologists. 
As a novel form of intervention, the Visual Perception Clinic's patient population and clinical impact are uncharacterised. Yet in an aging population with increasing coincidence of ophthalmic and neurodegenerative disease, the need for effective intervention is also increasing. It is therefore important to evaluate the impact of this clinic, which may provide a future model for the management of complex visual symptoms.

\section{Materials and methods}

We identified patients using the Eye Department outpatient's database. All patients attending the Visual Perception Clinic from June 2010 to February 2012 were used in the analysis. The data was collected by retrospective audit using Newcastle Hospitals electronic clinic summaries. Where electronic discharge summaries were unavailable, we used paper notes from Eye Department records. We have used representative case studies to illustrate the case mix seen and patient experiences in greater depth.

\section{Results}

We identified 50 patients between June 2010 and February 2012. Two patients had failed to attend, and for one no notes were obtainable. Therefore, 47 patients were included in the analysis. Mean age of patients was 76.2 (range 51-92) with slightly more women than men: 53\% (25/47) and 47\% (22/47), respectively.

The nature of presenting complaints is given in Table 1 . Almost half $(47 \%, 22 / 47)$ the patients attended with one main presenting complaint. An almost equal number $(49 \%, 23 / 47)$ had two or more presenting complaints; $26 \%(12 / 47)$ had two main complaints; $23 \%$ (11/47) had three or more. The remaining $4 \%(2 / 47)$ were asymptomatic, having been referred with abnormal examination findings. A significant number of patients had pre-existing neurological, ophthalmic, or psychiatric comorbidities (Table 1).

Before clinic, $72 \%(34 / 47)$ of patients had seen an ophthalmologist, of whom $17 \%(8 / 47)$ had also seen a neurologist, and 15\% (7/47) a psychiatrist. One person (2\%) had consulted all three with regard to their visual symptoms. Referrals to clinic were made primarily by ophthalmology $(57.4 \%, 27 / 47)$, followed by the family practitioner $(30 \%, 14 / 47)$, psychiatry $(9 \%, 4 / 47)$, and finally neurology $(4 \%, 2 / 47)$.

Cognitive assessment was clinically indicated in $43 \%$ (20/47) patients. Assessments included the mini-mentalstate examination (MMSE), ${ }^{15}$ the revised Addenbrookes cognitive examination, ${ }^{16}$ and the Montreal Cognitive assessment (MoCA). ${ }^{17}$ A significant number of patients
Table 1 Presenting complaints and comorbidities of patients attending Visual Perception clinic

\begin{tabular}{lc}
\hline & Number of patients (\%) \\
\hline Presenting complaint includes & \\
Visual Hallucinations & $22(46)$ \\
Double vision & $10(21)$ \\
Blurred vision & $7(15)$ \\
Visuo-spatial difficulties & $6(13)$ \\
Nonspecific visual difficulties & $18(38)$ \\
& \\
Pre-existing diagnoses include & \\
Neurological & $28(60)$ \\
Alzheimer's disease & $3(6)$ \\
Lewy body dementia & $1(2)$ \\
Other dementia (unspecified) & $6(13)$ \\
Parkinson's disease & $9(19)$ \\
Previous stroke or TIA & $4(9)$ \\
Other & $5(11)$ \\
& \\
Ophthalmic & $23(49)$ \\
Psuedophakia & $8(17)$ \\
Cataract & $14(30)$ \\
Age-related macular degeneration & $5(11)$ \\
Glaucoma & $1(2)$ \\
Diabetic retinopathy & $1(2)$ \\
Retinitis pigmentosa & $1(2)$ \\
Other & $4(9)$ \\
Psychiatric & $8(17)$ \\
Depression & $4(9)$ \\
Anxiety & $3(6)$ \\
Psychosis & $1(2)$ \\
\hline
\end{tabular}

It should be noted that as each patient may have multiple complaints and comorbidities the percentages do not total $100 \%$.

$(68 \%, 32 / 47)$ had a diagnosis of cognitive impairment. Of these, $50 \%(16 / 32)$ were new diagnoses.

After clinic attendance, a significant number of patients were given a new diagnosis. There was also an increase in the number of diagnoses with a neuropsychiatric component, and a concomitant decrease in the number of purely ophthalmological diagnoses (Table 2).

Brain imaging was performed as clinically indicated in $21 \%$ (10/47) patients; including computerised tomography (CT) $(6 \%, 3 / 47)$ and magnetic resonance imaging (MRI) $(15 \%, 7 / 47)$. Two patients $(4 \%)$ had a DatSCAN, which is a single photon emission CT (SPECT) scan carried out with the dopaminergic presynaptic ligand FP-CIT. This enables visualisation of the distribution of dopamine transportation in the striatum and is useful to aid diagnosis of conditions such as Lewy body dementia and Parkinson's disease. One patient (2\%) had a Tc-99m HMPAO SPECT perfusion scan. Tc-99m HMPAO is a well-established isotype useful for examining regional cerebral blood flow. 
Table 2 Diagnosis before and after clinic attendance

\begin{tabular}{|c|c|c|c|}
\hline & $\begin{array}{c}\text { Preclinic } \\
\text { attendance, } \\
n(\%)\end{array}$ & $\begin{array}{c}\text { Postclinic } \\
\text { attendance, } \\
n(\%)\end{array}$ & P-value \\
\hline Diagnosis for main complaint & $25(53)$ & $46(98)$ & $<0.001^{*}$ \\
\hline Ophthalmic diagnosis only & $4(9)$ & $3(6)$ & 1.000 \\
\hline $\begin{array}{l}\text { Neuropsychiatric component } \\
\text { to presentation }\end{array}$ & $19(40)$ & $42(89)$ & $<0.001^{*}$ \\
\hline
\end{tabular}

${ }^{*} P$-values $<0.05$ are considered significant.

${ }^{a}$ Calculated from McNemar's test.

Following clinic attendance, 43\% (20/47) patients were referred for continuing care to other clinics: $9 \%(4 / 47)$ to neurology, $4 \%(2 / 47)$ to ophthalmology, $11 \%(5 / 47)$ to memory clinic, $11 \%(5 / 47)$ to psychiatry, $6 \%(3 / 47)$ to optometry, and $2 \%(1 / 47)$ to ear nose and throat. Treatment was initiated in clinic for $36 \%(17 / 47)$ of patients: $13 \%(6 / 47)$ had a medication change, $4 \%(2 / 47)$ underwent ophthalmic surgery, 9\% (4/47) had optical correction, and $11 \%(5 / 47)$ received counselling or other social input.

\section{Case studies}

Patient 1 A 61-year-old female patient presented to her family practitioner in August 2010 with dizziness, blurring of vision, and seeing lights, with a normal ophthalmic examination. She was referred to a general eye clinic where no explanation was found for her symptoms. CT head was normal. She reattended the eye clinic in October 2010 with deteriorating night vision and intermittent diplopia, and was examined by a second consultant. She was found to have an incongruous left hemianopia and a provisional diagnosis of retinal dystrophy was made. However, she had a normal electroretinogram and normal retinal appearance.

She was referred to the Visual Perception clinic June 2011, where she described bumping into things on her left, struggling with direction sense and orientation difficulties. Cognitive test scores were 26/30 on MMSE and 71/100 on Addenbrooke's, with significant visuospatial deficit (Figure 1). MRI showed bilateral symmetrical posterior parietal and occipital atrophy. A provisional diagnosis of atypical Alzheimer's disease with a profound visual element was made. Tc-99m HMPAO SPECT perfusion scan showed moderate posterior hypoperfusion in the dorsal occipital lobe (Figure 2), in keeping with a diagnosis of posterior cortical atrophy (visual variant) Alzheimer's disease.

Patient 2 A 77-year-old female patient with a history of previous retinal detachment was seen by her family
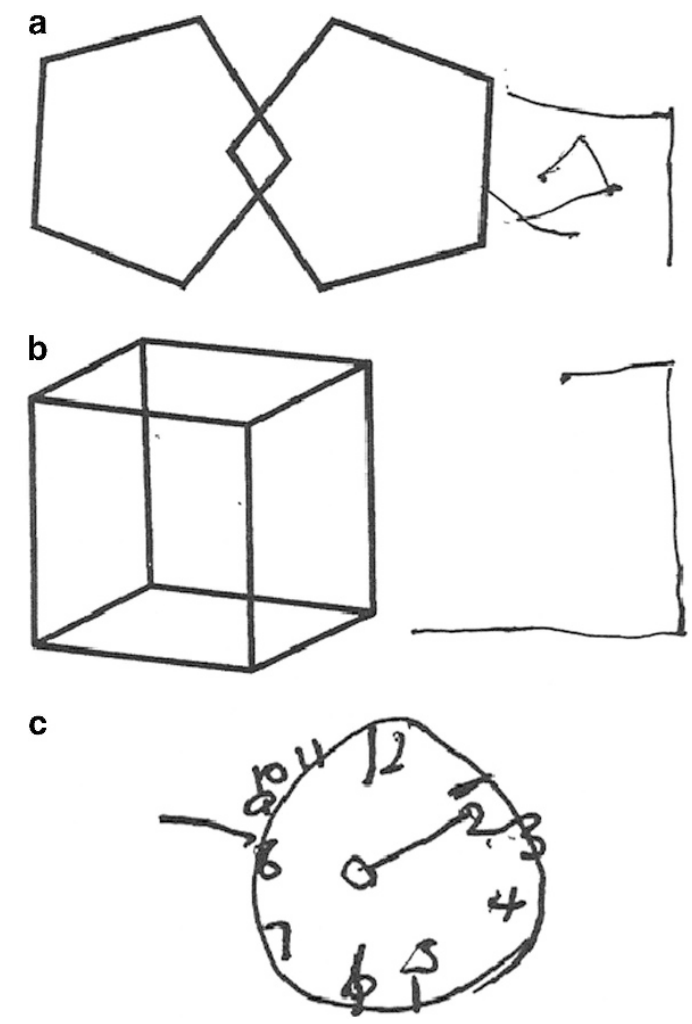

Figure 1 Tests of visuoconstruction from the Revised Addenbrooke's Cognitive Examination as performed by patient described in case 1: (a) Interlocking pentagons (Original left, patient copy right). (b) Necker cube (original left, patient copy right). (c) Clock drawing task. The patient was given instructions to draw a clock face with all the numbers and the hands at 10 past 5 . Note the uneven spacing of the numbers with many sitting outside the circle, and the absence of the hour hand.

practitioner complaining of seeing white lines and flowers. Her optician was unable to find an explanation for her symptoms but noted mild to moderate cataract. She was seen in the Visual Perception Clinic November 2010, where examination findings and CT scan were unremarkable and her visual acuities were $6 / 18$ on the right and 6/12 on the left. We reviewed her in March 2011 with worsening symptoms including right sided frontal-temporal headaches and patterned hallucinations. She showed cognitive impairment with a test score of $17 / 30$ on the MoCA. We arranged a DaTSCAN that showed reduced tracer uptake in the left putamen (Figure 3) consistent with probable dementia with Lewy Bodies. Her care is being continued under old-age psychiatry.

Patient 3 A 73-year-old male was referred by his optician late in 2005 with symptoms of a left visual field deficit. Humphreys and Goldmann field testing gave inconsistent results and he was referred to glaucoma clinic in early 2006. In 2007 he was referred with visual 


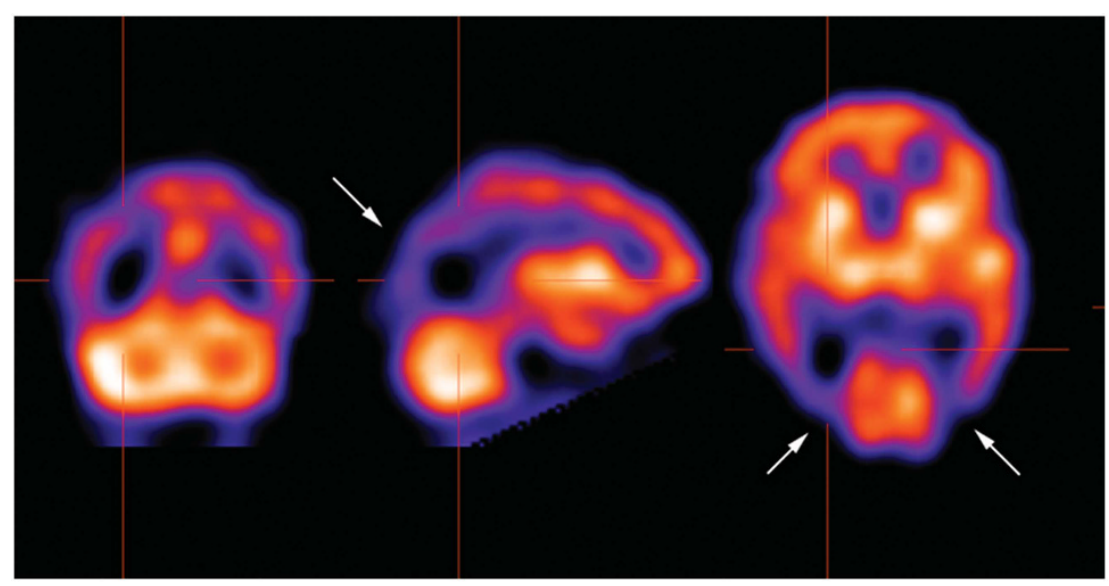

Figure 2 Case 1: Tc-99m HMPAO SPECT with coronal (left), sagittal (centre), and horizontal (right) sections. Bilateral parieto-occipital perfusion deficit which is slightly more marked in the right hemisphere (arrows). There is also some bilateral medial temporal hypoperfusion and left frontal hypoperfusion. The hypoperfusion is largely more posterior than anterior in keeping with the diagnosis of posterior cortical atrophy.

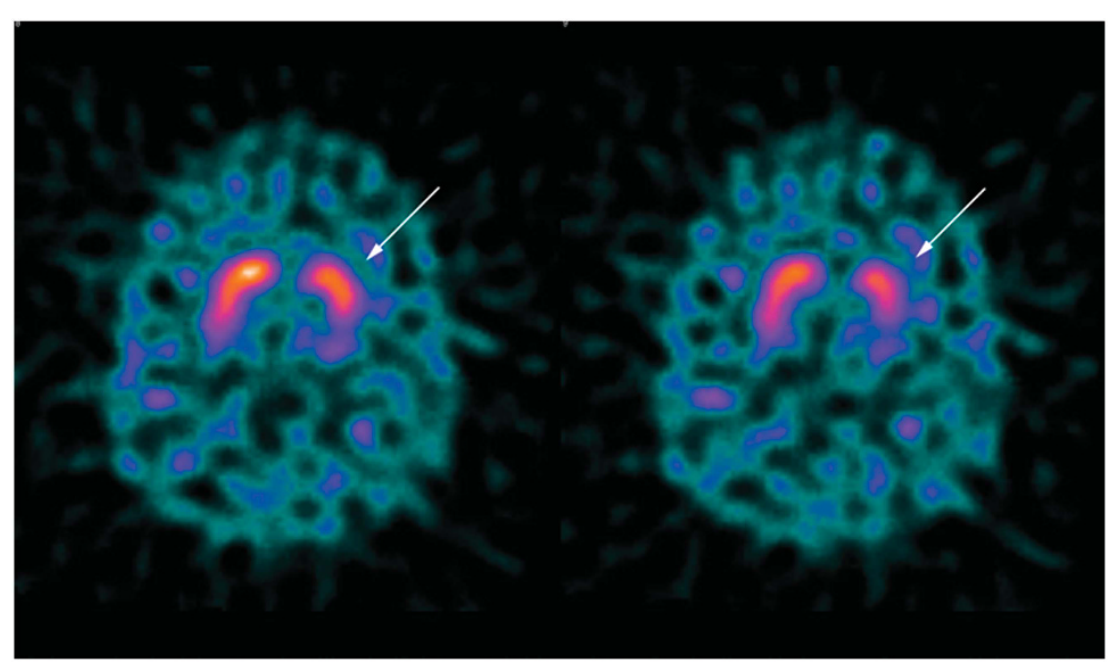

Figure 3 Case 2: FP-CIT presynaptic dopamine imaging scan (DaTSCAN). Horizontal sections showing reduced tracer uptake in the left putamen (arrow) consistent with the diagnosis of dementia with Lewy Bodies.

loss post total hip replacement, and examination revealed a right hemianopia. In 2008 and 2009, imaging performed for increasing forgetfulness showed a chronic left frontal infarct and generalised brain atrophy on CT and severe hypoperfusion in the left parieto-occipital, frontal, and medial temporal lobe on Tc-99m HMPAO SPECT perfusion imaging. He re-presented in 2010 to Neurology with visual hallucinations. In June 2011 he presented to general eye clinic with deteriorating visual acuity and continuing hallucinations. He was diagnosed with bilateral cataract and underwent left phaecoemulsification, but had no significant symptom relief.

In October 2011 he re-presented with deterioration of distance vision, losing his way around his home and difficulty in finding clothes and recognising faces, on a background of poor memory and continuing hallucinations. At this stage he was referred to Visual Perception Clinic. Examination revealed a right homonymous hemianopia, simultanagnosia, and central visual loss. A provisional diagnosis was made of vascular dementia with Balint's syndrome. The patient and his wife were counselled and indicated that despite a lack of management options, their primary motivation was to improve understanding of his symptoms. An MRI scan showed bilateral occipital atrophy. A Tc-99m HMPAO SPECT perfusion scan was therefore organised in order to look for any areas of hypoperfusion. This showed bilateral perfusion deficiencies in the occipital and parietal regions, right more severe than left. This pattern of bilateral, asymmetric cerebral hypoperfusion of the 
parieto-occipito regions is typical of visual variant Alzheimer's disease (posterior cortical atrophy).

\section{Discussion}

We have described our experiences of a novel, multidisciplinary visual perception clinic in the North of England. The population is mainly older people, with a high prevalence of visual comorbidities, and a significantly higher prevalence of neurological and cognitive impairment than the national average. $2,14,18$ Presentations were complex, with more than half presenting with multiple visual complaints. The most common symptoms were visual hallucinations and double vision. In the vast majority of cases patient symptoms could not be explained by eye pathology alone.

We have found that a combined clinic with neurological, ophthalmic, and psychiatric input is a highly effective method of diagnosing multifactorial, complex visual problems. The multi-disciplinary structure may be superior to a single physician or ophthalmologist working alone as it enables detailed, 'on the spot' evaluation of mental state and cognition as well as ophthalmic and neurological systems. The combined expertise of the team is likely to exceed that of an individual, particularly when considering dementia and psychiatric disease and interpreting imaging reports. Single specialists may underreport diagnoses outside their specialist area, which has been used as an argument to underscore the need for multi-disciplinary services for patients with cognitive complaints. ${ }^{19}$ Reviews of other multi-disciplinary clinics have found them to be an effective way of dealing with complex problems with multiple underlying aetiologies and simplifying the patient's diagnostic pathway. ${ }^{20-22}$ The case studies clearly illustrate that patients with atypical presentations of neurodegenerative disease with or without exacerbating concomitant eye pathology frequently visited many clinics before reaching a diagnosis. The aim of the Visual Perception Clinic is to prevent this continuing re-referral by acting as a one-stop service where comprehensive evaluation may be made and a representative diagnosis given. This is aided by multidisciplinary input, its location in Eye outpatients with ready access to investigative equipment, and clinic time available (40 min compared with the average Eye outpatient consultation time of $7 \mathrm{~min}$ ). Despite the requirements of a longer clinic time, we think this clinic model may ultimately reduce clinical burden by reducing time before diagnosis is made and preventing multiple, disconnected referrals to different specialties. Ophthalmologists and neuro-ophthalmologists may well be the best placed to lead such services, partly because they have the equipment required for ophthalmic assessment and partly because visual symptoms, whether due to ocular pathology or not, so often appear in ophthalmology clinics.

The results thus far indicate that the Visual Perception Clinic is a valuable resource that provides an effective service in integration of care. The case studies highlight the importance of thorough neurologic and neuropsychiatric evaluation in older people where visual impairment may be due to cerebral dysfunction. With an ageing population, and a rising prevalence of multifactorial visual problems, effective one-point access to multiple specialist inputs has become increasingly relevant. Owing to its relative novelty, only 47 patients have been evaluated in this study. The clinic clearly holds future potential for further research into its clinical impact, which may provide a model for the creation of similar clinics in other disciplines.

Summary

What was known before

- Eye pathology, psychiatric disease, and neurological conditions can all result in visual symptoms.

- Patients with complex visual symptoms inadequately explained by eye pathology alone can present a diagnostic challenge and often visit multiple specialty clinics before receiving a diagnosis.

What this study adds

- To our knowledge, this is the first description of a multidisciplinary clinic with neurology, psychiatry, and ophthalmology input for patients with complex visual symptoms.

- We describe the clinical presentations and outcomes from our early experience of this novel clinic, and present case studies to demonstrate how a multidisciplinary 'one-stop' clinic may provide high-quality care more effectively than multiple specialty appointments.

- We present the case that this clinic may act as a model for service provision in other ophthalmology departments or other specialties.

\section{Conflict of interest}

The authors declare no conflict of interest.

\section{Acknowledgements}

We thank Dr Jim Lloyd, Nuclear Medicine Department, Royal Victoria infirmary for his assistance with the images used in this paper. The research was supported by the National Institute for Health Research (NIHR) Newcastle Biomedical Research Centre based at Newcastle upon Tyne Hospitals NHS Foundation Trust 
and Newcastle University and by an NIHR Doctoral Research Fellowship award (2010-03-071 to JJ). The views expressed in this publication are those of the authors and not necessarily those of the NHS, the NIHR or the Department of Health.

\section{References}

1 Klaver CCW, Wolfs RCW, Vingerling JR, Hofman A, de Jong PTVM. Age-specific prevalence and causes of blindness and visual impairment in an older population. Arch Ophthalmol 1998; 116: 653-658.

2 Jackson GR, Owsley C. Visual dysfunction, neurodegenerative diseases, and aging. Neurol Clin 2003; 21 : 709-728.

3 Jefferis JM, Mosimann UP, Clarke MP. Cataract and cognitive impairment: a review of the literature. $\mathrm{Br} \mathrm{J}$ Ophthalmol 2011; 95: 17-23.

4 Jefferis JM, Mosimann UP, Taylor JP, Clarke MP. Do your eyes play tricks on you? Asking older people about visual hallucinations in a general eye clinic. Int Psychogeriatr 2011; 23: 1014-1015

5 Ong SY, Cheung CY, Li X, Lamoureux EL, Ikram MK, Ding J et al. Visual impairment, age-related eye diseases and cognitive function: the Singapore Malay eye study. Arch Ophthalmol 2012; 130: 895-900.

6 Manford M, Andermann F. Complex visual hallucinations Clinical and neurobiological insights. Brain 1998; 121: 1819-1840.

7 Galton CJ, Patterson K, Xuereb JH, Hodges JR. Atypical and typical presentations of Alzheimer's disease: a clinical, neuropsychological, neuroimaging and pathological study of 13 cases. Brain 2000; 123: 484-498.

8 Lee AG, Martin CO. Neuro-ophthalmic findings in the visual variant of Alzheimer's disease. Ophthalmology 2004; 111: 376-380.

9 Sanches-Ramos JR, Ortoll R, Paulson GW. Visual hallucinations associated with Parkinson Disease. Arch Neurol 1996; 53(12): 1265-1268.
10 Papapetropoulos S, Argyriou AA, Ellul J. Factors associated with drug-induced visual hallucinations in Parkinson's disease. J Neurol 2005; 252: 1223-1228.

11 Goetz G, Vogel C, Tanner CM, Stebbins GT. Early dopaminergic drug-induced hallucinations in parkinsonian patients. Neurology 1998; 51: 811-814.

12 Archibald NK, Clarke MP, Mosimann UP, Burn DJ. Visual symptoms in Parkinson's disease and Parkinson's disease dementia. Mov Disord 2011; 26: 2387-2395.

13 Brody BL, Gamst AC, Williams RA, Smith AR, Lau PW, Dolnak D et al. Depression, visual acuity, comorbidity, and disability associated with age-related macular degeneration. Ophthalmology 2001; 108: 1893-1900.

14 Clemons TE, Rankin MW, McBee WL. Cognitive impairment in the Age-Related Eye Disease Study: AREDS Report No 16. Arch Ophthalmol 2006; 124: 537-543.

15 Folstein MM, Folstein SE, McHugh PR. 'Mini-mental state'. A practical method for grading the cognitive state of patients for the clinician. J Psychiatr Res 1975; 12: 189-198.

16 Mioshi E, Dawson K, Mitchell J, Arnold R, Hodges JR. The Addenbrooke's Cognitive Examination Revised (ACE-R): a brief cognitive test battery for dementia screening. IN $t$ Geriatr Psychiatry 2006; 21: 1078-1085.

17 Nasreddine ZS, Phillips NA, Bédirian V, Charbonneau S, Whitehead V, Collin I et al. The montreal cognitive assessment, MoCA; a brief screening tool for mild cognitive impairment. J Am Geriatr Soc 2005; 53: 695-699.

18 Alzheimer's Association. 2012 Alzheimer's disease facts and figures. Alzheimer Dement 2012; 8: 131-168.

19 Verhey FR, Jolles J, Ponds RW, Rozendaal N, Plugge LA, de Vet RC et al. Diagnosing dementia: a comparison between a monodisciplinary and a multidisciplinary approach. J Neuropsychiatry Clin Neurosci 1993; 5: 78-85.

20 Mehta NR. Managing craniofacial pain the multidisciplinary way. Compend Contin Educ Dent 2012; 33: 224.

21 Vowles RH, Ghiacy S, Jefferis AF. A clinic for the rapid processing of patients with neck masses. J Laryngol Otol 1998; 112: 1061-1064.

22 Høgh P, Waldemar G, Knudsen GM, Bruhn P, Mortensen H, Wildschiødtz $\mathrm{G}$ et al. A multidisciplinary memory clinic in a neurological setting: diagnostic evaluation of 400 consecutive patients. Eur J Neurol 1999; 6: 279-288. 